\title{
HIGH-POWER RF TESTING OF A 352-MHZ FAST-FERRITE RF CAVITY TUNER AT THE ADVANCED PHOTON SOURCE*
}

\author{
D. Horan ${ }^{\#}$, E. Cherbak \\ Advanced Photon Source, Argonne National Laboratory \\ 9700 S. Cass Avenue, Argonne, IL 60439, U.S.A.
}

\begin{abstract}
A 352-MHz fast-ferrite rf cavity tuner, manufactured by Advanced Ferrite Technology, was high-power tested on a single-cell copper rf cavity at the Advanced Photon Source. These tests measured the fast-ferrite tuner performance in terms of power handling capability, tuning bandwidth, tuning speed, stability, and rf losses. The test system comprises a single-cell copper rf cavity fitted with two identical coupling loops, one for input rf power and the other for coupling the fast-ferrite tuner to the cavity fields. The fast-ferrite tuner rf circuit consists of a cavity coupling loop, a 6-1/8" EIA coaxial line system with directional couplers, and an adjustable $360^{\circ}$ mechanical phase shifter in series with the fast-ferrite tuner. A bipolar DC bias supply, controlled by a low-level rf cavity tuning loop consisting of an rf phase detector and a PID amplifier, is used to provide a variable bias current to the tuner ferrite material to maintain the test cavity at resonance. Losses in the fast-ferrite tuner are calculated from cooling water calorimetry. Test data will be presented.
\end{abstract}

\section{INTRODUCTION}

A $350-\mathrm{MHz}$ fast-ferrite tuner, manufactured by Advanced Ferrite Technology (AFT), was purchased by the Advanced Photon Source (APS) RF Group in 1996 for evaluation as an alternative for the slow motor-driven piston tuners used to maintain resonance in the APS storage ring room-temperature copper rf cavities. Lowpower rf tests on the fast-ferrite tuner were conducted in 2003 to determine the tuner's tuning range and speed [1]. At the completion of the low-power tests, the fast-ferrite tuner was prepared for high-power $\mathrm{CW}$ tests utilizing the APS 350-MHz RF test stand. Two separate high-power rf tests were performed, the first demonstrating the fastferrite tuner's ability to maintain resonance on a singlecell copper rf cavity at a maximum power level of $100 \mathrm{~kW} \mathrm{CW}$, and a second test where the fast-ferrite tuner was connected to the test stand rf input waveguide directly with no rf cavity in the system to test its power handling capability. The maximum power applied during the power-handling capability test was the specified 150$\mathrm{kW}$ maximum input power of the tuner. The fast-ferrite tuner was provided a DI water flow of 4.48 GPM and filtered dry-air flow for internal cooling of the bias coils and the rf structure, respectively. An arc detector was

\footnotetext{
*Work supported by U. S. Department of Energy, Office of Basic Energy Sciences, under contract No. W-31-109-ENG-38.

"horan@aps.anl.gov
}

optically coupled to the interior of the rf structure within the ferrite tuner that was configured to inhibit the applied rf power within $10 \mu$ s from the onset of arcing.

\section{CAVITY TUNING TESTS UP TO $100 \mathrm{~kW} \mathrm{CW}$}

The $100-\mathrm{kW}$ cavity tuning test system is shown in Figure 1. The cavity was fitted with two identical coupling loops, one for the cavity input power and one to couple the fast ferrite tuner to the cavity. Incident power was coupled to the cavity utilizing a WR2300 1/2-height waveguide, transitioning to a coaxial coupling loop at the cavity input port. The fast ferrite tuner was coupled to the cavity using 6-1/8" rigid coaxial line (required to mate with the input flange of the fast-ferrite tuner), which transitioned to the WR2300 1/2-height waveguide at the cavity-coupling loop [1]. A $360^{\circ}$ mechanical phase shifter, capable of operation at $150 \mathrm{~kW} \mathrm{CW}$, was part of the coaxial transmission line connecting the fast-ferrite tuner to the cavity and was used to vary the electrical length of the line to avoid resonance conditions, and to optimize the complex relationship between the tuning range of the fast-ferrite tuner and the test cavity input match. Also, two coaxial directional couplers were inserted in the 6-1/8" coaxial transmission system to provide the ability to monitor the coaxial line power levels. A conventional motor-driven piston tuner was installed in the cavity to provide sufficient tuning range to maintain resonance from a cold start. A standard analog cavity resonance control loop, consisting of a phase detector and PID amplifier, was used to control either the piston tuner position or the fast ferrite tuner bias current, which is variable from between $+100 \mathrm{~A}$ and $-100 \mathrm{~A}$. The maximum power level for this test is limited to $100 \mathrm{~kW}$ $\mathrm{CW}$, the input power limit of the test cavity and related components. The rate of power increase during the test was governed primarily by cavity vacuum conditions, due to re-conditioning required by the test cavity and related components after venting.

For the initial cavity tuning tests, the fast-ferrite coupling loop was set to the fully-horizontal (maximum coupling) position, which resulted in a non-matched condition between the loop and the cavity. The cavity input coupler was then set to the best $50-\Omega$ match possible with the fast-ferrite tuner network attached to the cavity, the $360^{\circ}$ mechanical phase shifter was set to approximately $45^{\circ}$, and zero bias current was applied to the fast-ferrite tuner. Under these conditions, the fastferrite tuner demonstrated an effective cavity tuning range 


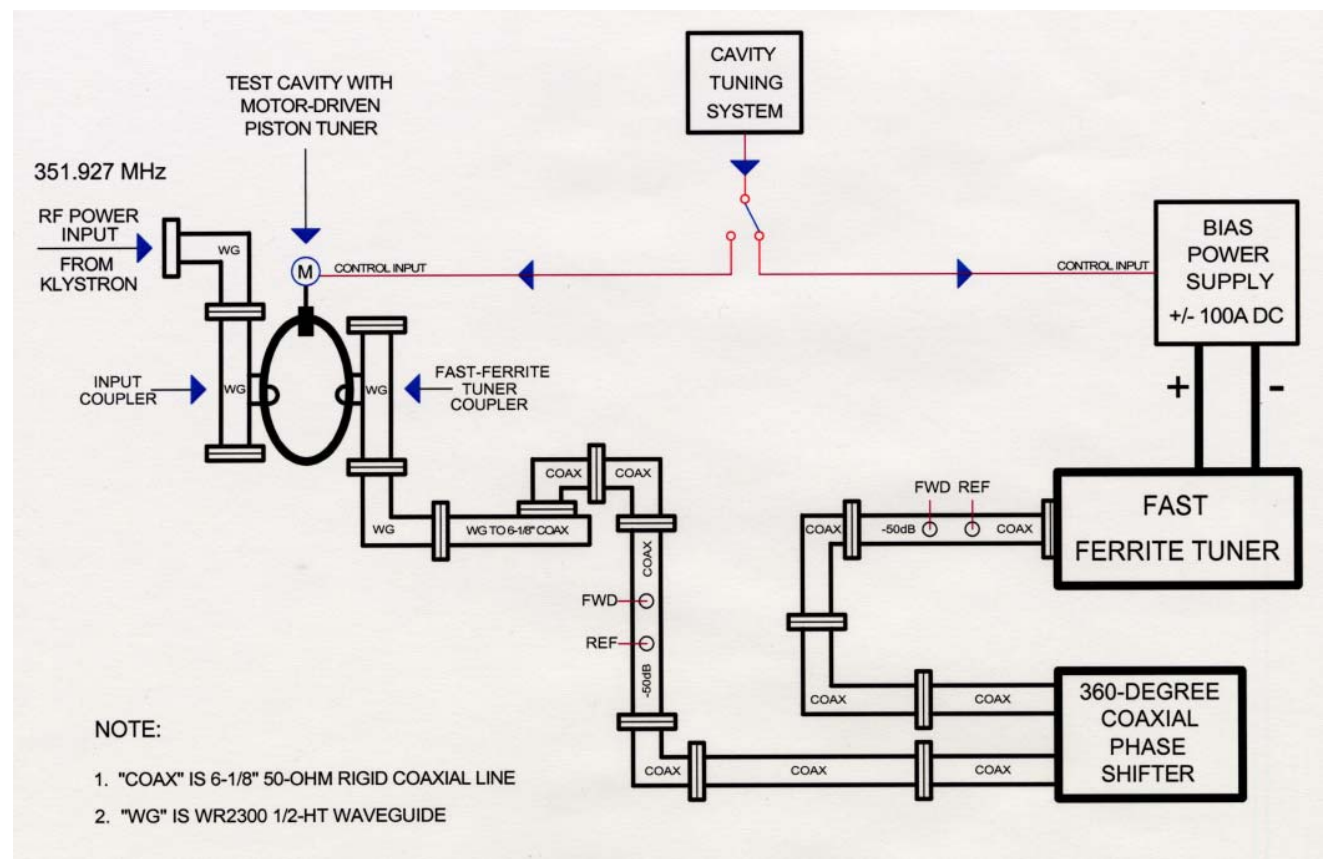

Figure 1: The 100-kW cavity tuning test system.

of approximately $110 \mathrm{kHz}$. However, the 6-1/8" coaxial line components in the test system exhibited evidence of overheating after operating the test system at approximately $42 \mathrm{~kW}$ for 12 hours. This overheating was due to extremely high voltage and current standing waves in the line, equivalent to a power level of approximately $142 \mathrm{~kW}$, which was measured using the coaxial directional coupler in line with the ferrite tuner. Air circulation was added to the coaxial line and mechanical phase shifter, but the temperature of the exhaust air from the line was still prohibitively high, reading approximately $71^{\circ} \mathrm{C}$ with a forward power of only $12.2 \mathrm{~kW}$ into the test cavity. The fast-ferrite tuner cavity coupler was then readjusted to a $5-\Omega$-matched position to reduce the amplitude of the voltage and current standing waves in the coaxial line. Figure 2 shows the relationship between the equivalent $\mathrm{rf}$ power level in the ferrite-tuner coupling line and the adjustment point of the $360^{\circ}$ mechanical phase shifter.

Initial tests were conducted at $10-\mathrm{kW}$ input power to detect the unfavorable line resonance conditions that can clearly be seen $180^{\circ}$ apart. The mechanical phase shifter was then adjusted to avoid overlapping the line resonance conditions over the $81^{\circ}$ operating range of the fast-ferrite tuner. Under these conditions, $100-\mathrm{kW}$ input power was achieved in the test cavity system with the fast-ferrite tuner maintaining cavity resonance, and power levels of $65 \mathrm{~kW}$ forward and $70.3 \mathrm{~kW}$ reflected were measured in the coaxial line between the fast-ferrite tuner and the cavity. The reduced coupling to the cavity lowered the internal temperature of the coaxial line to approximately $53^{\circ} \mathrm{C}$ at a cavity input power of $100 \mathrm{~kW}$, with the tradeoff being a reduced cavity tuning bandwidth of $16 \mathrm{kHz}$. Figure 3 shows the complex relationship between the fastferrite bias current, test cavity input return loss, and cavity resonant frequency at $100-\mathrm{kW}$ input power. The test system operated approximately 24 hours at power input levels of between $85 \mathrm{~kW}$ and $100 \mathrm{~kW}$ with no significant problems noted in the fast-ferrite tuner or related systems. There were two trips of the fast-ferrite tuner arc detector as the test system was conditioned to up to $100 \mathrm{~kW}$, but recovery from both trips was uneventful. The tuning speed of the fast-ferrite tuner was measured at a cavity input power of $50 \mathrm{~kW}$ and was found to be unchanged from identical measurements made under low-power conditions [1]. The rf power dissipated in the fast-ferrite tuner was derived from cooling water calorimetry and found to be less than 400 watts under all conditions.

After the test was completed and the test system dismantled, evidence of arcing was seen on the air-side of the cavity coupler and transition used to couple the fastferrite tuner to the cavity. This arcing, shown in Figure 4, was caused by over-voltage conditions generated by the extremely high-voltage standing-wave ratio in the fastferrite tuner transmission system. The arcing was not detected during system operation.

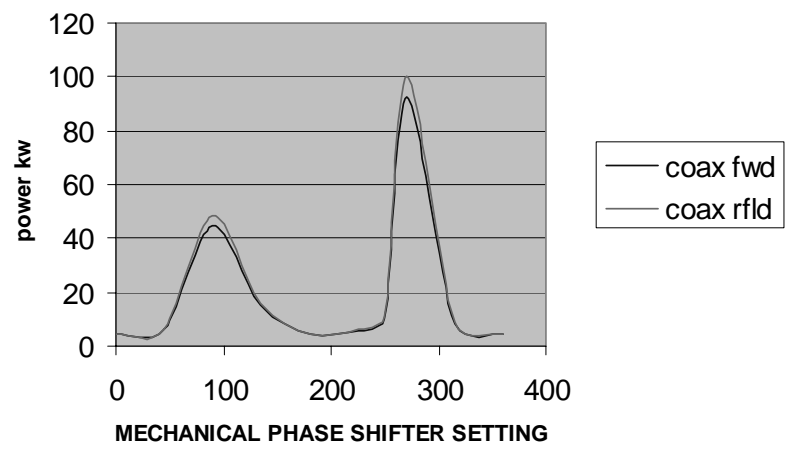

Figure 2: Resonance conditions in the fast-ferrite transmission system coaxial line. 


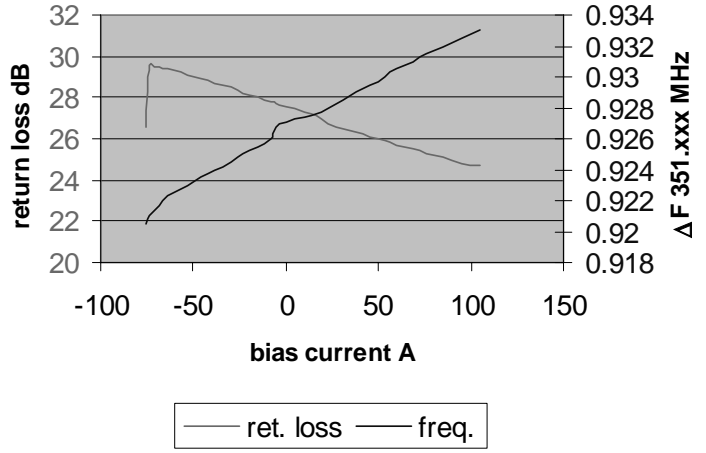

Figure 3: Relationship between fast-ferrite tuner bias current, cavity return loss, and resonant frequency.

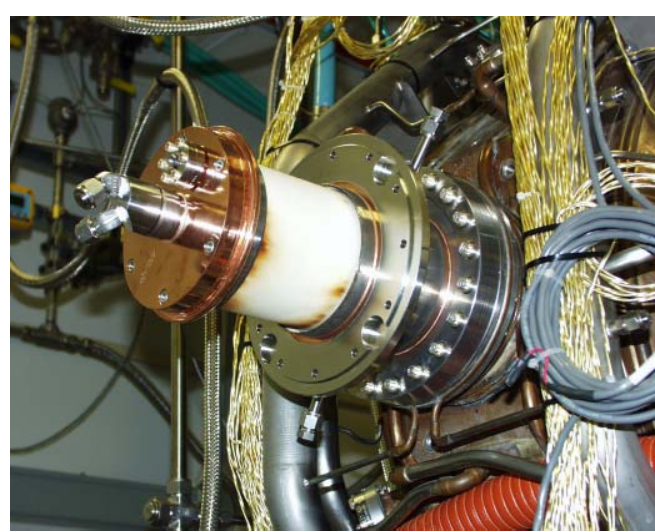

Figure 4: Arcing damage on cavity coupler transition.

\section{0-kW POWER HANDLING CAPABILITY TEST}

For this test, the fast-ferrite tuner and coaxial transmission system were connected to the test stand $\mathrm{rf}$ input waveguide directly, with no rf cavity in the system, to test the tuner under the maximum rf input power of $150 \mathrm{~kW}$ CW specified by AFT. The rf power reflected from the tuner was reflected back to the klystron output circulator, then dissipated in the circulator reject load. A vector-voltmeter was connected to the forward and reflected outputs of the coaxial directional coupler in-line with the fast-ferrite tuner to measure the phase shift generated by the tuner over the specified bias current range.

At power input levels of $10 \mathrm{~kW}, 50 \mathrm{~kW}$, and $100 \mathrm{~kW}$, the fast-ferrite tuner bias current was varied over the entire \pm 100 -A range in 10 -A steps. The tuner generated $81^{\circ}$ of phase shift at all power levels over the entire range of bias current. The resulting changes in reflection phase at port 1 of the klystron circulator caused the klystron output power to change in step with the tuner current. This effect was used to achieve $148 \mathrm{~kW}$ of forward power into the fast-ferrite tuner with a tuner bias current of $-100 \mathrm{~A}$. The power dissipation in the fast-ferrite tuner, calculated from cooling water calorimetry, was less than 400 watts ( \pm 118 watts) under all conditions. After approximately five minutes of operation at $148 \mathrm{~kW}$, a burning smell was detected in the area of the test stand bunker, so the test system was shut down for further investigation. After disassembly of the coaxial transmission system, evidence of arcing was discovered inside the $360^{\circ}$ mechanical phase shifter. Examination of the arcing pattern suggests that it was caused by overvoltage conditions, again brought about by the extremely high-voltage standing-wave ratio in the coaxial line. No other evidence of arcing was seen elsewhere in the coaxial system or in the fast-ferrite tuner.

\section{CONCLUSION}

The AFT 350-MHz fast-ferrite cavity tuner displayed the ability to maintain resonance of a single-cell rf cavity up to a power level of $100 \mathrm{~kW} \mathrm{CW}$, and to tune the cavity to resonance within 1 millisecond using a cavity tuning loop constructed with the standard APS rf system lowlevel rf components. The rf losses in the fast-ferrite tuner were less than 400 watts under all test conditions. Power handling limitations of the 6-1/8" coaxial transmission system required by the fast-ferrite tuner input flange significantly limited both the maximum power level and available tuning range of the tuner. Reliable use of this fast-ferrite tuner on the APS rf cavities at the $100-\mathrm{kW}$ rf power level would require significant hardware modifications, including supplemental cooling of the coaxial line, or possibly the elimination of the coaxial system by utilizing a waveguide transition to 6-1/8" EIA flange immediately adjacent to the input of the fast-ferrite tuner. Also, to optimize system reliability, a redesign of the standard APS cavity input coupler would be necessary in order for it to withstand the higher voltages encountered in the fast-ferrite tuner transmission system. A practical application of this fast-ferrite tuner would require that it be used in conjunction with a conventional motor-driven piston tuner to provide the tuning range necessary to maintain cavity resonance from cold-start to full-power conditions. In such an application, the fastferrite tuner would provide rapid cavity tuning to compensate for beam loading effects, while the piston tuner would be used to compensate for long-term thermally induced effects on resonant frequency.

\section{ACKNOWLEDGEMENTS}

The authors would like to thank Advanced Ferrite Technology for their valuable technical assistance in preparation for these tests, and also Jack Albert, Dave Meyer, Mike Drackley, Dave Bromberek, John Pace, and Andre McKenzie for their efforts in assembly of the test system.

\section{REFERENCE}

[1] D. Horan, E. Cherbak, "Fast-Ferrite Tuner Operation on a 352-MHz Single-cell RF Cavity at the Advanced Photon Source," PAC 2003, Portland, OR, May 1216, 2003, pp. 1177-1179, http://www.jacow.org. 